\title{
Exact, polynomial, determination solution method of the subset sum problem
}

\author{
Mahammad Maharram Aliyev \\ National Academy of Sciences of Azerbaijan, Institute of Control Systems, Baku, Azerbaijan \\ Email address: \\ aliyev.mahammad@yahoo.com
}

\section{To cite this article:}

Mahammad Maharram Aliyev. Exact, Polynomial, Determination Solution Method of the Subset Sum Problem. Applied and Computational Mathematics. Vol. 3, No. 5, 2014, pp. 262-267. doi: 10.11648/j.acm.20140305.21

\begin{abstract}
In this paper we give original geometrical interpretation to the domain of definition of integer and combinatorial problems. The solution of the problems concerning NP class has been carried out on the hyperarches. The existence criterion of the solution on the hyperarches has been defined. The method for establishing the sequence of approximation to the solution on the hyperarches was constructed. Calculation experiments were conducted, and the obtained polynomial algorithm, practically and theoretically solved exactly the (SSP) problem.
\end{abstract}

Keywords: Knapsack Problem (KP), Subset Sum Problem (SSP), NP Class, Integer Programming, N-Dimensional Cube, Hyper Plane, Hyper Circle, Hyper Arch

\section{Introduction}

The problems in computing mathematics are divided into two classes according to their difficulty: 1) the first class contains the problems in which the number of simple operations for its solution (addition, subtraction, division, comparison, and square root) is polynomial expressed by the parameters (by the number of $n$-variables, the number of $m$-restriction) of the problem.

This class in said to be a polynomial class and in short is called a $P$ class. 2) The problems in which the number of simple operations for its solution is proportional to $2^{n}$ enter to $N P$ class. This class is called NP hard class. The knapsack problem is one of the classic problems in the linear integer programming problems. Up to present time, it was not possible to solve this problem exactly by a polynomial algorithm.

The knapsack problem can be formulated as a solution of the following linear integer programming formulation:

$$
\begin{aligned}
& (K P) \text { maximize } \sum_{j=1}^{n} p_{j} x_{j} \\
& \text { subject to } \sum_{j=1}^{n} w_{j} x_{j} \leq c
\end{aligned}
$$

$$
x_{j} \in\{0 ; 1\} \quad j=1,2, \ldots, n
$$

We will denote the optimal solution vector by $X^{*}=\left(x_{1}^{*}, x_{2}^{*}, \ldots, x_{n}^{*}\right)$ and the optimal solution value by $Z^{*}$. The set $X^{*}$ denotes the optimal solution set, corresponding to the optimal solution vector.

Problem $(K P)$ is the simplest non-linear integer programming model with binary variables, only one single constraint and only positive coefficients. Nevertheless, adding the integrality condition (1.3) to the simple linear program (1.1)-(1.2) already puts $(K P)$ into the class of "difficult" problems [1].

The knapsack problem has been studied for centuries as it is the simplest prototype of a maximization problem. Already in 1897 Mathews showed how several constraints may be aggregated into one single knapsack constrain. This is somehow a prototype of a reduction of a general integer program to $(K P)$, thus proving that $(K P)$ is at least as hard to solve as an integer program. It is however unclear how the name "Knapsack Problem" was invented. Dantzig is using the expression in his early work and thus the name could be a kind of folklore [1].

When $p_{j}=w_{j}$ in $(K P)$, the resulting optimization problem is known as the subset sum problem (SSP) because we are looking for a subset of the values $w_{i}$ with the sum being as close as possible to, but not exceeding the given target value $c$. 


$$
\begin{gathered}
(S S P) \text { maximize } \sum_{j=1}^{n} w_{j} x_{j} \\
\text { subject to } \sum_{j=1}^{n} w_{j} x_{j} \leq c \\
x_{j} \in\{0,1\}, j=1, \ldots, n
\end{gathered}
$$

Although (SSP) is a special case of $(K P)$ it is still $N P$-hard and is $N P$ - complete [1].

The class of $N P$-complete problems $N P C$ is the set of decision problems $Q$ satisfying the following two properties:

$$
\begin{gathered}
Q \in N P \\
\forall R \in N P: R \leq_{p} Q
\end{gathered}
$$

To see the latter, one may simply assume that an $N P$-complete problem $Q$ could be solved in polynomial time, then by transforming $R$ to $Q$ we would also get a polynomial algorithm for $R$ contradicting the assumption.

The theory of $N P$-completeness gives us a framework for showing that it is very doubtful that a polynomial algorithm for solving e.g. the subset sum problem, then we would also be able to solve numerous famous optimization problems like the travelling salesman problem, general integer programming, and we would even be able to efficiently find mathematical profs of theorems, as stated in Cook [1].

In this paper, a new solution method of knapsack problem is given by an original approach; some problems are solved exactly by a polynomial algorithm. One is subset sum problem.

\section{Problem Statement}

At first the engineers and economists has been engaged integer problems. These problems appeared as a result of economic and engineering demands. Therefore, these problems have got engineering and economic interpretation. But these interpretations are not enough for the solution of the problem.

In order to solve any mathematical problem, it is necessary to give its geometrical interpretation. It is the work of professional mathematicians. To this end, this matter requires special attentive in this paper.

We consider that, reader no need to use any references by reading the paper.

When constructing the new method we'll take three principles:

1. Definition of existence criterion of the solution integer point in the considered domain;

2. Construction of the sequence approximating to the solution using the found point;

3. Definition of necessary and sufficient conditions for the found solution to be optimal.

\section{Investigation of $n$-Dimensional Cube}

$\mathrm{S}$ is the set of vertex points of $n$-dimensional cube whose rib equals a unit, one of the vertexes is on the origin of coordinate, the ribs originate from the origin of coordinates and are arranged on the coordinate axes. The set is equal to the $\operatorname{set} x_{j} \in\{0 ; 1\}, j=1,2, \ldots, n \quad$ [2].

Let's investigate the structure of $n$-dimensional cube and mathematical relations between its elements. When a rib of $n$-dimensional cube equals a unit, the coordinates of its vertex points consists of a unit and zero. The number of these vertices is $S=\sum_{k=0}^{n} C_{n}^{k}=2^{n}$. For the indicated $k$ the $C_{n}^{k}$ number points are such that their $k$ number coordinates consist of a unit, $n-k$ number coordinates consist of zero. The equation of planes holding these points is as follows:

$$
x_{1}+x_{2}+\cdots+x_{n}=k ; \quad k=\overline{1, n-1}
$$

It is seen from equation (3.1) that the planes holding the $C_{n}^{k}$ number integer points are parallel to each other and these plane equations change only depending on the free term $k$ in the right hand side. Denote these hyper planes by $M_{n k}$. $R_{k}=\sqrt{k}$ is the distance of these points from the origin. $d_{k}=\frac{k}{\sqrt{n}}$ is the distance of hyper planes $M_{n k}$ from the origin, $o_{n k j}=\frac{k}{n} ; j=\overline{1, n}$ is the center of a hyper circle located on hyper planes $M_{n k}$ and holding the vertices of $n$-dimensional cube, contained in $M_{n k} \cdot r_{n k}=\sqrt{\frac{n k-k^{2}}{n}}$ Is a radius of this hyper circle? Denote by $K_{n k}$ a hyper circle of a radius $r_{n k}$ and center at the point $O_{n k}$ located on the hyper plane $M_{n k}$. On each $n$-dimensional space we can group the set $S$ being the vertices of $n$-dimensional cube with a rib equal a unit on parallel hyper planes $M_{n k}$, we solve problem $(K P)$ on each $K_{n k}$ and find general optimal solution. It is a general geometric principle. By solving the optimization problem considered in any domain we solve the problem in the parts whose unions equal to the domain and intersections are empty sets, find optimal solution at each part, compare them and then find general optimal solution.

\section{Correlation of Constraint Hyper Plane and Hyper Circles}

Let's study correlations of the constraint plane $M:\left\{\sum_{j=1}^{n} w_{j} x_{j}=c\right\}$ and hyper circle $K_{n k}$. Let's normalize the vector $W\left(w_{1}, w_{2}, \ldots w_{n}\right)$ and denote it by $W_{n} .\left|W_{n}\right|=1$ Find the projection of the vector $W$ on the hyper plane $M_{n k}$ and denote it by $W_{p}$ 


$$
W_{p}=W_{n}-\left(W_{n}, N_{n}\right) \cdot N_{n} .
$$

$N_{n}\left(\frac{1}{\sqrt{n}}, \frac{1}{\sqrt{n}}, \cdots, \frac{1}{\sqrt{n}}\right)$ are the normal of the hyper plane $M_{n k}$. One of the three relations between the hyper plane $M$ and hyper circles $K_{n k}$ is possible:

1) $c>\left(W, D_{1}\right)$ (Figure 1:Illustration of the correlation of constraint hyper plane and hyper circles). Here $D_{1}$ is the point obtained from the intersection of the straight line passing through the point $O_{n k}$ in the direction $W_{p}$ and the hyper circle $K_{n k}$ and giving the maximum value to the inner product $(W, X)$ on $K_{n k}$. In this case, any point lying on $K_{n k}$ satisfies the constraint: $\sum_{j=1}^{n} w_{j} x_{j} \leq c$.

2) $c<\left(W, D_{2}\right)$. Here the points $D_{2}$ is the point obtained from the intersection of the straight line passing through the point $O_{n k}$ in the direction $W_{p}$ and giving the minimal value to the inner product $(W, X)$ on the hyper circle $K_{n k}$. In this case none of the points located on the $K_{n k}$ satisfies the constraint: $\sum_{j=1}^{n} w_{j} x_{j} \leq c$.

3) $\left(W, D_{2}\right) \leq c \leq\left(W, D_{1}\right)$. In this case either the hyper plane $M$ intersects the hyper circle $K_{n k}$ or touches it at the points $D_{1}, D_{2}$. In order to solve problem $(K P)$ we solve it on each hyper circle $K_{n k} \subset M_{n k}$ and denote these solutions by $X_{n k}^{*}$.

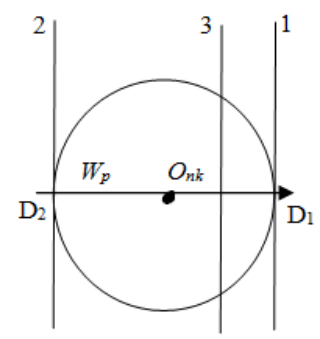

Figure 1. Illustration of the correlation of constraint hyper plane and hyper circles

$$
\left.\begin{array}{c}
\max _{S}\left(\sum_{j=1}^{n} p_{j} x_{j}\right)=\max _{k}\left(\sum_{j=1}^{n} p_{j} x_{n k j}^{*}\right) \\
\sum_{j=1}^{n} p_{j} x_{j} \leq c \\
\sum_{j=1}^{n} x_{j}=k \\
\sum_{j=1}^{n}\left(x_{j}-o_{n k j}\right)^{2}=r_{n k}^{2}
\end{array}\right\}
$$

In the first case the solution of the problem $(K P)$ on the Knk is found as follows. We give unit values to $x_{j}$ corresponding to $k$ number $p_{j}$ taking the greatest value.

To other $n-k$ number $x_{j}$ we give zero value.

In the second case the problem has no solution on $K_{n k}$. In this case we don't look for the solution on $K_{n k}$ and $M_{n k}$, respectively.

In the third case the solution of problem $(K P)$ on $K_{n k}$ is of special importance. The general solution of the problem reduces to its solution in the third case [3]. In the considered case, the domain for the solutions of the problem is a hyper arch being a part of a hyper circle. We express the hyper arch analytically in the following way

\section{Criterion on the Existence of Solution Points on a Hyper Arch}

In order to solve the problem we have to define the criterion on the existence of integer points on a hyper arch. By $X(n, k)$ we denote a set of integer points contained in the hyper circle $K_{n k}$ whose $k$ number coordinates equal a unit, $n-k$ number coordinates equal a zero. The hyper plane $M:\left\{\sum_{j=1}^{n} w_{j} x_{j}=c\right\}$ intersects the hyper circle lying on $M_{n k}$ and divides it into two parts (figure 2: Illustration of the criterion on the existence of solution points on a hyper arch).

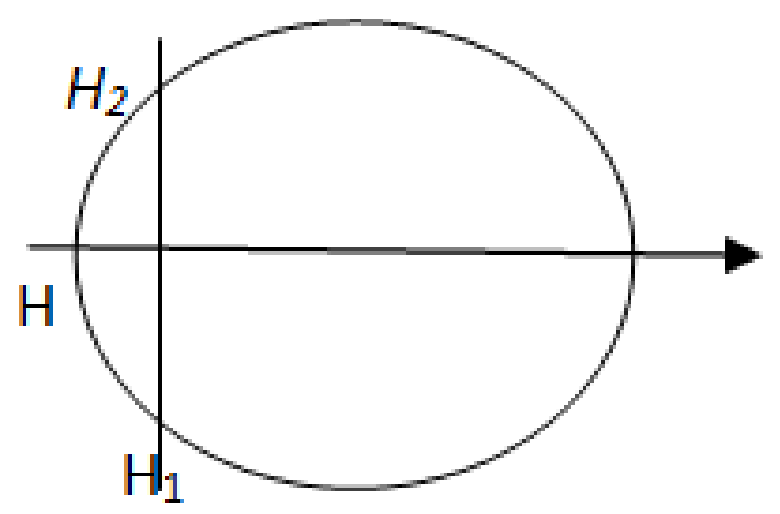

Figure.2. Illustration of the criterion on the existence of solution points on a hyper arch

The hyper arch $H_{1} H_{2}$ satisfies the constraint $\sum_{j=1}^{n} w_{j} x_{j} \leq c$.

Let's give the criterion on the existence of even if one or none

of these points on this hyper arch.

For this we find the $k-t h$ minimum element among the elements of the vector $W$ and denote it by $w_{k m}$. We give unit value to the $x_{1 j}$ corresponding to the $k$-number $w_{j}$ satisfying the condition $w_{k m}-w_{j} \geq 0$, the zero value to other $n-k$ number $x_{1 j}$. Let's verify if the point $X_{1}(n, k)$ 
obtained in such a way satisfies the constraint $\sum_{j=1}^{n} w_{j} x_{j} \leq c$. If the point $X_{1}(n, k)$ doesn't satisfy the constraint the problem $(K P)$ has no solution in $K_{n k}$ and $M_{n k}$ respectively.

If $X_{1}(n, k)$ satisfies the constraint of problem $(K P)$ has a solution in $K_{n k}$ and $M_{n k}$, respectively. $X_{1}(n, k)$ is a point or one of the points contained in $X(n, k)$ and giving the minimum value to the inner product $(W, X)$.

So, we defined the criterion on the existence of solution point on a hyper arch [3].

\section{The Local Property Any Point Lying on the Hyper Circle}

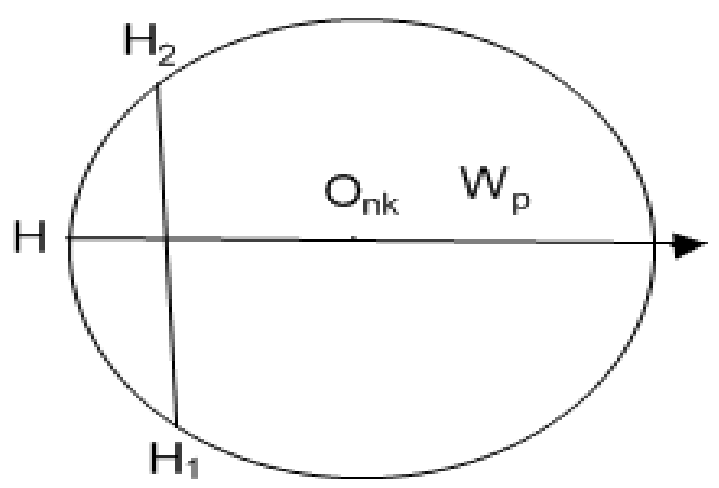

Figure. 3. Illustration of the local property any point lying on the hyper circle

Now let's consider the vector $\mathrm{HO}_{\text {nk }}$ (fig. 3: Illustration of the local property any point lying on the hyper circle). Let's define relation between coordinates of the vector $\mathrm{HO}_{\mathrm{nk}}$ and initial point of this vector $\mathrm{H} . \mathrm{H}\left(\mathrm{h}_{1}, \mathrm{~h}_{2}, \ldots, \mathrm{h}_{\mathrm{n}}\right)$; Onk $\left(\frac{k}{n}, \frac{k}{n}, \ldots, \frac{k}{n}\right)$, $j=\overline{1, n}$. By hoj we denote the coordinates of the vector $\mathrm{HO}_{\mathrm{nk}}$. $\mathrm{h}_{\mathrm{oj}}=\frac{k}{n}-\mathrm{h}_{\mathrm{j}}, \mathrm{j}=\overline{1, n}$.

Here the maximum values of hj correspond to the minimal of hoj. As hoj consist of the difference of the constant quantity $\frac{k}{n}$ and hj values

If we consider the vector $\mathrm{HO}_{\mathrm{nk}}$ as a normal of a plane and take the minimal $\mathrm{k}$ number hoj and give to corresponding $\mathrm{x}_{1 \mathrm{j}} \mathrm{a}$ unit and the others $n-k$ number $x_{1 j}$ zero values we can determine the point $\mathrm{X} 1(\mathrm{n}, \mathrm{k}) \in \mathrm{X}(\mathrm{n}, \mathrm{k})$ or one of these points close to $\mathrm{H}$ in the vicinity of the point $\mathrm{H}$. This is a local property of any point lying on the hyper circle $\mathrm{K}_{\mathrm{nk}}$.

In future we'll use this property in constructing the sequence of approximating to solution of integer points on a hyper arch.

\section{The Construction of a Sequence Converging to the Solution on a Hyper Arch}

Let's give analytic expression of the problem on a hyper arch:

$$
\begin{aligned}
& \sum_{j=1}^{n} p_{j} x_{j} \rightarrow \max \\
& \sum_{j=1}^{n} w_{j} x_{j} \leq c \\
& \sum_{j=1}^{n} x_{j}=k \\
& \sum_{j=1}^{n}\left(x_{j}-o_{n k j}\right)^{2}=r_{n k}^{2} \\
& x_{j} \in\{0 ; 1\}
\end{aligned}
$$

Assume that the point $X_{1}(n, k)$ is determined by the existence criterion and is located on the hyper circle $K_{n k}$. We use this point and construct a sequence converging to the solution of problem (7.1). The terms of the sequence should satisfy the constraint and give strong monotonically increasing values to the functional.

1) Monotony condition: take the first of the coordinates equal zero of the point $X_{1}(n, k)$ and compare it with the corresponding coordinates of the point $X_{1}(n, k)$ taking unit values $p_{i}$ and $p_{j} . p_{i}-p_{j}>0$.

2) Constraint satisfaction condition. Let's give constraint satisfaction condition for the indices $i$ and $j$ satisfying the monotony condition $p_{i}-p_{j}>0$. We calculate the quantity $d=c-\sum_{j=1}^{n} w_{j} x_{1 j} ; d+w_{j}-w_{i} \geq 0$ are constraint satisfaction condition.

$$
\left\{\begin{array}{l}
p_{i}-p_{j}>0 \\
d+w_{j}-w_{i} \geq 0
\end{array}\right.
$$

If condition (7.2) is satisfied, we accept $x_{1 j}=0 ; x_{1 i}=1$. The point $X_{2}(n, k)$ found in such a way increases the functional and satisfies the constraint and is located on $K_{n k}$.

We continue this process until the relation (7.2) is not satisfied between the indices $i$ and $j$.

We conduct the process $k=\overline{1, n-1}$ for all $K_{n k}$ to $k=\overline{1, n-1}$ find the points $X_{q}(n, k)$ compare the values that they give to the functional and find the solution of problem $(K P)$

$$
Z^{*}=\max _{k}\left(P, X_{q}^{*}(n, k)\right) ; k=\overline{1, n} ; P\left(p_{1}, p_{2}, \ldots, p_{n}\right) \text { is object }
$$
vector.

\section{Necessary and Sufficient Condition of the Integer Point Lying on the Hyper Arch to be Local Optimal Solution on a Hyper Arch}

Denote by A the negation of condition (7.2).

Theorem: The necessary and sufficient condition for the integer point on the edge hyper arch to be local optimal 
solution, is condition A between coefficients $\mathrm{pj}$ and wj according to $x_{j}=1$ coordinates and coefficients pi and wi according to $\mathrm{x}_{\mathrm{i}}=0$ coordinates.

Necessities: Suppose that the point $X^{*}(n, k)$ is a local solution point on the edge hyper arch, on the hyper circle $\mathrm{K} \mathrm{n}$ $\mathrm{k}$.

$$
\max (\mathrm{P}, \mathrm{X}(\mathrm{n}, \mathrm{k}))=\left(\mathrm{P}, \mathrm{X}^{*}(\mathrm{n}, \mathrm{k})\right)
$$

Assume contrary. If the point $\mathrm{X}^{*}(\mathrm{n}, \mathrm{k})$ does not satisfy condition $\mathrm{A}$, when there exist the coordinates $\mathrm{x}_{\mathrm{j}}=1$ and $\mathrm{x}_{\mathrm{i}}=0$ such that $p_{i}-p_{j}>0$. If we accept $x_{j}=0$ and $x_{i}=1$ then we can find the point $\bar{X}(\mathrm{n}, \mathrm{k})$ such that

$\left(\mathrm{P}, \mathrm{X}^{*}(\mathrm{n}, \mathrm{k})\right)<(\mathrm{P}, \bar{X}(\mathrm{n}, \mathrm{k}))$

$\mathrm{Z}\left(\mathrm{X}^{*}(\mathrm{n}, \mathrm{k})\right)<\mathrm{Z}(\bar{X}(\mathrm{n}, \mathrm{k}))$

This contradicts the local optimal solution of the point $X^{*}(n$, $\mathrm{k})$. The obtained contradiction prove the necessities.

Suffices: Assume that the point $X^{*}(n, k)$ satisfies the condition A. Prove that this point is a local optimal solution on a hyper arch. Prove that is a maximum value of the functional on the edge of the hyper arch $Z^{*}=\left(P, X^{*}(n, k)\right)$. Assume contrary. $Z^{*}$ is not a maximum value of the functional on the edge of the hyper arch. Here is a point $\bar{X}(\mathrm{n}, \mathrm{k})$ such that $\bar{Z}=$ $(\mathrm{P}, \bar{X}(\mathrm{n}, \mathrm{k}))$ is maximum value of the functional. Show that in this case the point $X^{*}(n, k)$ do not satisfy the condition $A$.

$\mathrm{k}$ number non zero coordinates of the point $X^{*}(\mathrm{n}, \mathrm{k})$ denote by $\mathrm{x}_{11}, \mathrm{x}_{12}, \ldots, \mathrm{x}_{\mathrm{lk}}$. By $\mathrm{x}_{\mathrm{q} 1}, \mathrm{x}_{\mathrm{q} 2}, \ldots, \mathrm{x}_{\mathrm{qk}}$ denote non zero coordinates at the point $\bar{X}(\mathrm{n}, \mathrm{k})$. Then

$$
\begin{gathered}
\mathrm{Z}^{*}=\mathrm{p}_{11}{ }^{*}+\mathrm{p}_{12} *+\ldots+\mathrm{p}_{\mathrm{lk}} * \quad \mathrm{p}_{\mathrm{lj}}>0 ; \mathrm{j}=\overline{1, k} \\
\bar{Z}=\bar{p}_{\mathrm{q} 1}+\bar{p}_{\mathrm{q} 2}+\ldots+\bar{p}_{\mathrm{qk}} \bar{p}_{\mathrm{q} j}>0 ; \mathrm{j}=\overline{1, k} \\
\text { If } \bar{Z}>\mathrm{Z} * \text { hens } \\
\bar{p}_{\mathrm{q} 1}+\bar{p}_{\mathrm{q} 2}+\ldots+\bar{p}_{\mathrm{qk}}>\mathrm{p}_{11} *+\mathrm{p}_{12} *+\ldots+\mathrm{p}_{\mathrm{lk}} *
\end{gathered}
$$

There is $\bar{p}_{q j}>p^{*}{ }_{l j}$; $l j q j$ such that $\bar{p}_{q j}-p^{*}>0$. This fact contradicts fulfillment of the condition $A$ at the point $X^{*}(n, k)$. As the obtained contradiction, contradicts the theorems condition. Suffices is proved.

\section{Estimation of the Number of Iterations and its Decrease}

Now estimate the number of iterations. If any point $X_{l}(n, k)$ is an initial point, then the number of iterations is

$$
\mathrm{N}_{\mathrm{it}} \leq \mathrm{c}-\left(\mathrm{W}, \mathrm{X}_{1}(\mathrm{n}, \mathrm{k})\right)
$$

$p_{i^{-}} p_{j} \geq 1$ at each iteration, the value of the functional increases at last by a unit. Find a closer initial point to hyper plane $(W, X)=c$, we can provide the condition $N_{i t} \leq n$. In order to solve these problem, we must chose the integer $c_{l}$ that satisfies condition $\left(W, X_{1}(n, k)\right)<c_{1}<c$ close to $c . c-c_{l}<n$. Find the intersection point of the hyper plane $(W, X)=c_{1}$ and the straight line $W_{\min } W_{\max }$ and denote it by $T$ (Fig.4: Illustration of the decrease number of iterations). Then we must find the vector $\mathrm{L}$ parallel to the intersection of hyper planes $(W, X)=c$ and $x_{1}+x_{2}+\ldots+x_{n}=k$. Transfer in parallel way the point $T$ to on the hyper circle $K_{n k}$, in the direction of $L$ and $-L$ and find the points $H_{1}$ and $H_{2}$ on the hyper circle $K_{n k}$. Show these operations concretely.

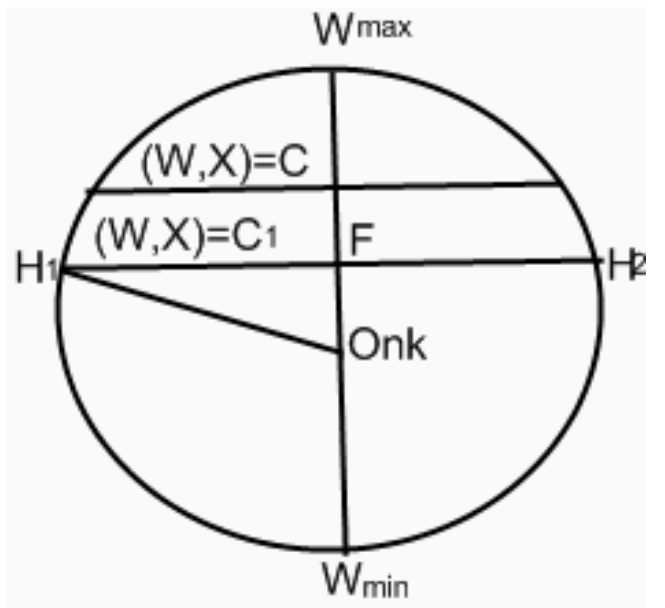

Figure 4. Illustration of the decrease number of iterations

Normalize the vector $W$ and denote it by $W_{n}$. Find the projections of this vector on the hyper plane $M_{n k}$.

$$
\mathrm{W}_{\mathrm{p}}=\mathrm{W}_{\mathrm{n}}-\left(\mathrm{W}_{\mathrm{n}}, \mathrm{E}_{\mathrm{n}}\right) \mathrm{E}_{\mathrm{n}} ; \mathrm{E}_{\mathrm{n}}=\left(\frac{1}{\sqrt{n}}, \frac{1}{\sqrt{n}}, \ldots, \frac{1}{\sqrt{n}}\right)
$$

Normalize the vector $W_{p}$ and denote it by $W_{p n}$. Transfer in a parallel way the point $O_{n k}$ by $r_{n k}$ in the direction of $W_{p n}$ and $-W_{p n}$. Denote the obtained points by $W_{\max }$ and $W_{\min } \cdot W_{\max }=O_{n k}$ $+r_{n k} W_{p n} ; W_{\text {min }}=O_{n k}-r_{n k} W_{p n}$.

Find the intersection points of the straight line passing through the point $W_{\min }$ in the direction $W_{p n}$ and the hyper plane $(W, X)=c_{l}$ and denote it by $\mathrm{T}$.

$$
\mathrm{T}=\mathrm{W}_{\min }+\left[\left(\mathrm{c}_{1}-\left(\mathrm{W}, \mathrm{W}_{\min }\right)\right) /\left(\mathrm{W}, \mathrm{W}_{\mathrm{pn}}\right)\right] \mathrm{W}_{\mathrm{pn}}
$$

Find the vector $L$ parallel to the intersection of the hyper plane $w_{1} x_{1}+w_{2} x_{2}+\ldots+w_{n} x_{n}=c_{1}$ and hyper plane $x_{1}+x_{2}+\ldots$ $+x_{n}=k$.

$$
\left\{\begin{array}{c}
(W, L)=0 \\
(E, L)=0
\end{array}\right.
$$

Here $E=(1,1, \ldots, 1)$. When the dimension of the space is $n>3$ the problem has infinitely number solutions.

$$
\left\{\begin{array}{c}
w_{1} l_{1}+w_{2} l_{2}+\cdots+w_{n} l_{n}=0 \\
l_{1}+l_{2}+\cdots+l_{n}=0
\end{array}\right.
$$

In order to solve the system (9.1) we use the following principle. Chose $w_{k} \neq w_{l}$ and give the values $l_{j}=p_{j}$ to the elements of the vector in the direction $L$ different from the elements $j \neq 1 ; j \neq k$. The determinant of the obtained system of equation is

$$
\mathrm{D}=\left|\begin{array}{cc}
w_{1} & w_{k} \\
1 & 1
\end{array}\right|=w_{1-} w_{k}
$$

This system always has solution. 


$$
\begin{gathered}
\left\{\begin{array}{c}
w_{1} l_{1}+w_{k} l_{k}=s_{1} \\
l_{1}+l_{k}=s_{2}
\end{array}\right. \\
s_{1}=-\sum_{j=1}^{n} w_{j} p_{j}+w_{1} p_{1}+w_{k} p_{k} \\
s_{2}=-\sum_{j=1}^{n} p_{j}+p_{1}+p_{k} \\
l_{1}=\frac{s_{1}-w_{k} s_{2}}{w_{1}-w_{k}} ; l_{2}=\frac{w_{1} s_{2}-s_{1}}{w_{1}-w_{k}}
\end{gathered}
$$

Thus we determined the $L I I\left(M \cap M_{n k}\right)$ direction vector. Normalize the vector $L$ and denote it by $L_{n}$.

$$
\begin{gathered}
\mathrm{d}=W_{\text {min }} F \text { (figure) } \\
\mathrm{d}=\frac{c_{1}-\left(W, W_{\text {min }}\right)}{\left(W, W_{p n}\right)} \\
d_{1}=O_{n k} F=I r_{n k}-d I \\
d_{2}=F H_{1}=F H_{2} \\
d_{2}=\sqrt{r_{n k}^{2}-r_{n k}^{2}+2 r_{n k} d-d^{2}}=\sqrt{2 r_{n k} d-d^{2}} \\
\mathrm{H}_{1}=\mathrm{W}_{\text {min }}+\mathrm{dW}_{\mathrm{pn}}+\mathrm{d}_{2} \mathrm{~L}_{\mathrm{n}} \\
\mathrm{H}_{2}=\mathrm{W}_{\text {min }}+\mathrm{dW}_{\mathrm{pn}}-\mathrm{d}_{2} \mathrm{~L}_{\mathrm{n}}
\end{gathered}
$$

Find the points $X_{1}(n, k)$ and $X_{2}(n, k)$ close to the points $H_{1}$ and $\mathrm{H}_{2}$ and from these points construct a sequence approximating to the solution of the both of ends of the hyper arch. Compare the points according to the value given to the functional and find the local solution on the hyper circle $X^{*}(n, k)$.

Apply these processes for all the hyper circle for the $k=\overline{1, n}$. $\bar{X}(\mathrm{n}, \mathrm{k})$ satisfying condition

$$
\mathrm{Z}=\max \left(\mathrm{W}, \mathrm{X}^{*}(\mathrm{n}, \mathrm{k})\right) ; \mathrm{k}=\overline{1, n} .
$$

will be an optimal solution of the problem.

It showed be noted that while pasting to other end, the following principle may be as well.

If any point $X_{I}(n, k)$ on the hyper arch is known, we find intersection hyper plane $(W, X)=\left(W, X_{l}(n, k)\right)$ and the line $W_{\min } W_{\max }$ and denote it by $T$. Then transfer in a parallel way the point $T$ on the hyper circle in the direction of the vector $L=$ $\overrightarrow{X_{1}(n, k) T} \cdot X_{2}(n, k)=T+L$.

Calculation experiments given in [4].

\section{Investigation of Difficulty Order of the Problem}

To understand difficulty order we must consider the cycles contained in each other in the alqorithm:

$$
k=\overline{1, n-1} ; j=\overline{1, n} ; i=\overline{1, n} ; k k=\overline{1, n} ;
$$

The maximal number of embedded cycles is four.

Hence we proved polynomial property of the method and that its difficulty order is $O\left(n^{4}\right)$.

\section{Conclusion}

In this work we obtained the following new results.

1) Geometric interpretation of domain of definition of the solution of combinatorial and discrete problems is given.

2) A method for reducing the solution of any linear integer programming problem to its solution on a hyper arch is given.

3) Existence criterion of this problem on a hyper arch is defined.

4) Using the initial point found on a hyper arch a method for constructing the sequence approximating to the solution is given.

5) The difficulty order of the algorithm is a polynomial.

6) Necessary and sufficient conditions for the found point to be an optimal solution on the edge of hyper arch is given. This condition is analytic.

7) Obtained method is exact, polynomial and determination of the subset sum problem (SSP).

\section{References}

[1] Hans Kellerer, Ulrich Pferschy, David Pisinger Knapsack Problems. Spinger-Verlag Berlin. Hidelberg, 2004, 525 p.

[2] Мину М. Математические программирование. Москва, Наука 1990, 485 с.

[3] Aliyev M.M. One approach to the solution of the knapsack problem//Reports NAS of Azerb., 2005, №3, p.32-39.

[4] Aliyev M.M. On Solution Method of the Knapsack Problem. Proceedings of the Sixth International Conference on Management Science and Engineering Management. Volume I, Springer-Verlag, London, 2013, p.257-267. 